\title{
ELECTIONS TO MEMBERSHIP
}

\section{Ordinary Membership}

Abramson, Fred G, U of Wis-Milwaukee, Milwaukee, WI Abu-Said, Ahmed M, Ga Tech, Atlanta, GA Ackler, Lynn, Fitchburg St C, Fitchburg, MA Addington, Susan L, SUNY, Ctr at Stony Brook, Stony Brook, NY Aggarwal, Shiv Kumar, 209-A Branson St, Chapel Hill, NC Airault, H, NYU-Courant, New York, NY Alder, Hermann C, U de Concepcion, Concepcion, Chile Aldrich, Roger W, Perkin-Elmer, Norwalk, CT Aleskeviciene, A K, Lithuanian Acad of Sci, Vilnius, U S S R Amadio, William J, Rider C, Lawrenceville, NJ Ambasht, Jamuna Prasad, Voorhees C, Denmark, SC Anderson, Bertil, 23 Parkwoods Rd, Plandome, NY Anderson, Peter O, UCLA, Los Angeles, CA Anderson, Philip H, Montclair St C, Upper Montclair, NJ Andrews, H Robert, Prairie St C, Chicago Heights, IL Appelson, Edward Harvey, Optical Clinic, Dallas, TX Aschbacher, Michael, Caltech, Pasadena, CA Asibong, Ufot I, U of Waterloo, Waterloo, Ontario Can Auspitz, Norman E, IBM Canada, Toronto, Ontario Can Aytch, Annas, U of Pittsburgh, Pittsburgh, PA

Badescu, Lucian, $U$ of Bucharest, Bucharest, Rumania Banica, Constanin, Inst of Math, Bucharest (12), Rumania Barry, Hubert G, Jacksonville St U, Jacksonville, AL Baruch, Herbert M Jr, 840 Napoli Dr, Pacific Palisades, CA Bazelow, Alexander Rudolf, Polytech Inst of New York, Farmingdale, NY Ben-Jacob, Marion Glazerman, 63-50 Wetherole St, Rego Park, NY

Beniger, James R, UCB, Berkeley, CA

Berestycki, Henri, U of Chicago, Chicago, IL Berger, Agnes, Columbia U, New York, NY Berline, Steven M, US Air Force, Scott AFB, IL Bernardo, Jose M, U of Valencia, Valencia (10), Spain Bethel, James S, US Army, White Sands MR, NM Bieler, Richard K, Lake Tahoe Commun C, South Lake Tahoe, CA Blake, Michael G, Mich St U, East Lansing, MI Blankonship, Gilmer L, Case Western Reserve U, Cleveland, $\mathrm{OH}$ Boback, Kenneth G, 59 E Kirmar Ave, Nanticore, PA Bodfish, Edward R Jr, 1082 Hyde Park Ave, Boston, MA Bouffard, Alan J, 713 College Ave, Pittsburgh, PA Boyd, Eddie Jr, Okla St U, Stillwater, OK Brannigan, Michael, $U$ of Natal, Durban, So Africa Brewer, Dennis William, U of Ark, Fayetteville, AR Brooks, Roy G, U of Tex at Permian Basin, Odessa, TX Brown, R E, Globe Wholesale, Itasca, IL Bunce, Gary R, Winona St U, Winona, MN Burge, Calvin K, Greenville C, Greenville, IL Butler, Helen I, Cal St U Hayward, Hayward, CA Byerly, Howard L, Belen Consolidated Schools, Belen, NM Cantu, Mariajose M, 9120 Bridgewater St, College Park, MD Capizzi, Thomas, Temple U, Philadelphia, PA Carlson, James A, U of Utah, Salt Lake City, UT Carro, Anthony, Federal Aviation Agcy, Atlantic City, NJ Carter, James W, P O Box 8332, San Marino, CA Cashman, William T, C of Charleston, Charleston, SC Castore, Glenn M, 2027 E Genesee, Apt 19, Syracuse, NY Ceriani, William J, Pa Bureau of Employment Security, Altoona, PA 
Chakrabarti, S, UCD, Davis, CA

Chambliss, Josephine, Oxford Schools, Oxford, MS

Charalambus, Demetrois Thomas; Motorola, Franklin Place, IL

Charoennibhonvanich, Napaporn, Yeshiva U, New York, NY

Cheng, Sui-Sun, UCD, Davis, CA

Chosid, Leo, New York City Commun C (CUNY), Brooklyn, NY

Chung, Fan R K, Bell Tel Labs, Murray Hill, NJ

Cochell, Gary G, 20-9 N University PI, Stillwater, OK

Cogosvili, George, Acad of Sci, Tbilisi (Georgia), U S S R

Cohen, Daniel Isaac, Northeastern U, Boston, MA

Coman, Gheorghe, Fac Math, Cluj (Napoca), Rumania

Commins, William R, State of Cal, San Francisco, CA

Cotiu, Aurel, Inst Politehnic, Cluj (Naroca), Rumania

Cross, Ronald W, U of Cape Town, Rondebosch (CP), So Africa

Cruikshank, Joel R, 911 E Bridger, Pocatello, ID

Culik, Karel, U of Mass, Amherst, MA

Cullinane, Patricia Annmarie, 13 Bow St, Wellesley, MA

Cullinane, Steven, 134 Huxley St, Jamestown, NY

Curry, Robert L, Cal St U Fullerton, Fullerton, CA

Dasaro, George Earl, 1302 S General McMullen, San Antonio, TX

Dazord, Jean M, U of Lyon, (69621) Villeurbanne, France

De Sautel, David L, Saginaw Valley St C, University Center, MI

Dean, Alice, U of Mass, Amherst, MA

Demetropoulos, Andrew, Montclair St C, Upper Montclair, NJ

Dempsey, Francis E Jr, IBM, Kingston, NY

Dennis, Emmett C, Western Mich U, Kalamazoo, MI

Dennis, John L, Wright St U, Dayton, OH

Di Cenzo, Alan, 301 S Wayne St, Arlington, VA

Dobkin, David P, Yale U, New Haven, CT

Dooley, A H, Australian Natl U, Canberra (Act 2600), Australia

Douthat, Sybil G, P O Box 593, Toano, VA

Duncan, Ernest R, Rutgers U, New Brunswick, NJ

Dunn, Gerald J III, U of New Orleans, New Orleans, LA

Dunn, Joseph C, Cornell U, Ithaca, NY

Dunn, Mark R, Thiokol, Brigham City, UT

Durand, Loyal, U of Wis, Madison, WI

Durrett, Richard T, UCLA, Los Angeles, CA

Dyce, Byron A, 2629 NW 55th Blvd, Gainesville, FL

Edwards, William Lee, Computer Sci, San Diego, CA

Edwards, William R Jr, U of Southwestern La, Lafayette, LA

Elliott, J E H, Open U, Milton Keynes, England

Elosser, Paul Douglas, U of Ky, Lexington, KY

Ephraim, Robert M, Herbert H Lehman C (CUNY), Bronx, NY

Epperson, James F, Carnegie-Mellon U, Pittsburgh, PA

Esakia, L L, Acad of Sci, Georgian SSR, (380086) Tbilisi, U S S R

Evans, Lawrence Craig, U of Ky, Lexington, KY

Ezra, Paul, 4127 37th St, San Diego, CA

Fagedes, Thomas $\mathrm{P}$, Xavier U, Cincinnati, OH

Farley, Laura E, Fordham U, Bronx, NY

Feigilis, Robin, Yale U, New Haven, CT

Finlay, Doris Ruopp, US Environ Protection Agcy, Washington, DC

Fisher, Doreen R, Santa Rosa Jr C, Santa Rosa, CA

Fitting, Marjorie A, San Jose St U, San Jose, CA

Fleming, Clark, Va Highlands Commun C, Abington, VA

Flynn, Robert, Polytech Inst of New York, Brooklyn, NY

Foldes, Stephane, U of Waterloo, Waterloo (N2L3G1), Ontario Can

Fowler, Mary E, Control Data, Sunnyvale, CA

Fraser, Beverly A, Stevens Inst of Tech, Hoboken, NJ

Freund, T G, CMMS, Inc, Plainfield, NJ

Frickel, Loren I, P O Box 1033, Manchester, CT

Furst, Merrick L, Cornell U, Ithaca, NY

Gaffney, Terence James, Brown U, Providence, RI

Galperin, Efim A, 4615 Bourret Ave, Apt 10, Montreal, Quebec Can

Garay, Eduardo, P O Box 133, Cambridge, MA 
Garrison, Lionel B Jr, Dwight Englewood School, Englewood, NJ Gavin, Lloyd A, Cal St U Sacramento, Sacramento, CA Gegelia, Tengiz G, Math Inst, Tbilisi (93), U S S R Gerisch, Wolfgang, Laubenheinerstr 33, 1 Berlin 33, Germany Gerlach, Walter P, Southeast Commun C, Cumberland, KY Gettens, Daniel J, Rensselaer Polytech Inst, Troy, NY Giammerse, Jack, LSU, Baton Rouge, LA Ginsburg, Karen Halbert, C of Mt St Vincent, Riverdale, NY Glessner, William W, B7 Student Village, CWSC, Ellensburg, WA Golland, Louise F, 10510 S Hamilton, Chicago, IL Goller, Richard T, Edgecliff C, Cincinnati, OH Gomez, Jose A, $715 \mathrm{~W}$ Madison St, 4, Ann Arbor, MI Goodstein, Mitchell J, 1849 W 3rd Pl, Mesa, AZ Gordon, C M, U of Cambridge, Cambridge, England Gorin, Allen L, Bronx Commun C (CUNY), Bronx, NY Grammatikopoulos, Myron K, U of Ioannina, Ioannina, Greece Grand, Jeremy H, 601 Estancia, Caparra Heights, Rio Piedras, PR Gray, Robert M, Stanford U, Stanford, CA Gresser, John T, Bowling Green St U, Bowling Green, $\mathrm{OH}$ Gromov, Mikhael, SUNY, Ctr at Stony Brook, Stony Brook, NY Gruber, George M, 5713 12th Ave, Brooklyn, NY Guerrero, Ignacio, Brandeis U, Waltham, MA

Gulati, Chandar, Escola Graduada, (01000) Sao Paulo, Brazil Guzek, Joseph A, Hughes Aircraft, Los Angeles, CA Haagensen, Kenneth E, 1918 Jefferson St NE, Minneapolis, MN Haboush, William J, Inst for Adv Study, Princeton, NJ

Hailpern, Brent, Stanford U, Stanford, CA

Hamberg, Charles L, Adlai E Stevenson HS, Prairie View, IL

Hancock, Timothy L, Rice U, Houston, TX

Hansen, Harry E Jr, Esterline Elec, Costa Mesa, CA

Harris, John G, Northwestern U, Evanston, IL

Harris, R Michael, Vought, Dallas, TX

Hassinger, Bill Jr, Hassinger Wholesale, Greensboro, NC

Hasz, Earl E, Metropolitan St C, Denver, CO

Haug, David R, U of Colo, Boulder, CO

Hawkins, John B, NCR, Jackson, MS

Haynes, Robert D, Presearch Inc, Arlington, VA

Hays, M M R, 86 Crossbrook Rd, Amherst, MA

Healey, Paul, Detroit Inst of Tech, Detroit, MI

Heckl, Joseph P, US Naval Ordinance Lab, Silver Spring, MD

Heines, Carol G, Tex Southern U, Houston, TX

Henderson, Gregory P, U of South Fla, Tampa, FL

Henry, Charles Francis T, Rt 2, Box 18L, Gambrills, MD

Heppa, Douglas V, 77-38 79th St, Glendale, NY

Herman, Joan E, Yale U, New Haven, CT

Hernandez-Lerma, Onesimo, Brown U, Providence, RI

Heron, G H, Anoka-Ramsey St Commun C, Coon Rapids, MN

Herzog, Emil R, Cal St Polytech U, Pomona, CA

Hink, Robert E, J F Hink \& Son, Berkeley, CA

Holt, William R, Bookringer Ingelheim Ltd, Elmsford, NY

Houghton, Robert, 136 Chatfield Rd, Bronxville, NY

Huang, Peter H, Harvard U, Cambridge, MA

Huang, Yean-Ming, U of S C, Aiken, SC

Huddleston, Philip L, Parks C of Aeronautical Tech, Cahokia, IL Hughes, Anne, St John's U, Jamaica, NY

Hughes, Rhonda J, Tufts U, Medford, MA

Hundhausen, Joan Rohrer, Colo School of Mines, Golden, CO

Huszar, Miklos, Hungarian Acad of Sci, (114) Budapest, Hungary

Idowu, Elayne A, U of Pittsburgh, Pittsburgh, PA

Ifcher, Ruth S, 175-14 W 87th St, New York, NY

Ihrig, E C, McMaster U, Hamilton, Ontario Can

Ismail, Mourad E H, McMaster U, Hamilton, Ontario Can

Ivery, John J, Ala St U, Montgomery, AL

Jacobs, Elliott, Adelphi U, Garden City, NY 
Janecke, James R, Upper Iowa U, Fayette, IA

Jason, Gary, 21165 Escondido St, Woodland Hills, CA

Jen, Cornelius C, U of III, Urbana, IL

Johnson, Colonel Jr, Southern U, Baton Rouge, LA

Johnson, Mark A, Upjohn, Kalamazoo, MI

Johnson, Roy K, 4308 C Boxwood Ct, Huntsville, AL

Johnston, Katherine G, Vanderbilt U, Nashville, TN

Jones, John A, NSF, Washington, DC

Jones, Robert C, Southwest Tex St U, San Marcos, TX

Joseph, James E, Federal City C, Washington, DC

Joshi, Dhananjay S, Ill Inst of Tech, Chicago, IL

Kaman, Charles H, Digital Equipment, Maynard, MA

Kardas, Don, 2331 Miller, Ann Arbor, MI

Kasper. Toni. Borough Manhattan Comm C (CUNY), New York, NY

Keane. William J, U of Notre Dame, Notre Dame, IN

Kenderov, P S, Bulgarian Acad of Sci, (1000) Sofia, Bulgaria

Kent, William H, Stevens Inst of Tech, Hoboken, NJ

Kerwin, Sister Carolyn, Marygrove C, Detroit, MI

Khan, Mohammad Ahmad, Kuwait U, Kuwait

Khosrovshahi, G B, U of Tehran, Fac of Sci, Tehran, Iran

Kioustelidis, John, Tech U of Athens, Athens (809), Greece

Klamka, Jerzy, ul Andrzeja $29 \mathrm{~m} \mathrm{6,(40-061)} \mathrm{Katowice,} \mathrm{Poland}$

Klein, Albert J, Youngstown St U, Youngstown, OH

Kleinschmidt, Peter, Ruhr Univ, (4630) Bochum, Fed Rep Germany

Knaus, Nadine L, U of Tex at Austin, Austin, TX

Knowles, Wendy M, TRW, Redondo Beach, CA

Kockinos, Constantin N, 2121 Creeden Ave, Mountain View, CA

Kohlmayr, Gerhard F, Mathmodel Cons Bureau, Glastonbury, CT

Kornblum, Deborah, Brooklyn C (CUNY), Brooklyn, NY

Kosmala, Witold A J, U of South Fla, Tampa, FL

Kowol, Gerhard, U of Vienna, Math Inst, Vienna (A 1090), Austria

Kozlowski, George, LSU, Baton Rouge, LA

Kruelle, Mark F, Cornell U, Ithaca, NY

Kuku, A O, U of Ibadan, Ibadan, Nigeria

Kulvicki, Dennis M, U of Tex at Austin, Austin, TX

Kurosaka, Robert T, Mass Bay Commun C, Watertown, MA

Kutateladze, Semen, Zolotodolinskaja 9 FL14, Novosibirsk (630072), U S S R

Kwok, Paul K T, Southern Conn St C, New Haven, CT

Labbiento, Giovanni, U de Montreal, Montreal (H1Z2N6), Quebec Can

Ladd, H O Jr, 319 16th St, Virginia Beach, VA

Lagrange, R H, U of Wyo, Laramie, WY

Lai, Laurence, 1709 E 15th St, Brooklyn, NY

Lambert, John P, Ballistic Res Lab, Aberdeen Proving Ground, MD

Lance, Timothy L, SUNY, Ctr at Albany, Albany, NY

Larkin, James, 132 Old Sib Rd, Ridgefield, CT

Laughlin, Lester H, Lockheed MSC, Sunnyvale, CA

Lazza, Jon A, RFD 1, Wharton, $\mathrm{OH}$

Legg, David A, Indiana-Purdue U, Ft Wayne, IN

Leiber, Franklin H, SUNY, Ctr at Stony Brook, Stony Brook, NY

Lenahan, Terrence A, Bell Labs, Norcross, GA

Lenker, Terry D, U of Louisville, Louisville, KY

Lev, Joseph, 53 Royal Blvd, Delmar, NY

Levin, Vladimir L, Acad of Sci, CEMI, Moscow (117333), U S S R

Lin, Chia-Shiang, U of Ill, Urbana, IL

Lin, James P, UCSD, La Jolla, CA

Lindley, Thomas L III, Rockwell Internatl, Los Angeles, CA

Lipps, Frederick Weissner, U of Houston, Houston, TX

Lipton, Maurice, US Govt, Washington, DC

Liss, Frederick, U of Wis-Wausau, Wausau, WI

Livsic, Moshe S, TEVZ, Kvartal 106, Korpus 1, KV37, (380078) Tbilisi, U S S R

Lobello, Anthony, Box 181, Gambier, OH

Lohuis, David J, U of Va, Charlottesville, VA

Looney, Carl G, U of Toledo, Toledo, $\mathrm{OH}$ 
Low, Susan, Armijo Adult School, Fairfield, CA

Lubliner, Irving M, UCD, Davis, CA

Lucow, William H, Rider C, Lawrenceville, NJ

MacDonald, Carolyn Trott, Baptist $\mathrm{C}$ at Charleston, Charleston, SC

MacKey, Thomas S, Key Metals \& Minerals Engr, Texas City, TX

MacKichan, Barry, N M St U, Las Cruces, NM

Mager, Peter S, MRI Sys, Austin, TX

Mahar, T, NYU-Courant, New York, NY

Majthay, Elizabeth, U of Fla, Gainesville, FL

Makarov, V L, B L Ukrainki 24, AP 68, Kiev (133), U S S R

Malcev, Ju N, Altai St U, Barnaul (65099), U S S R

Maleki, Pir M, Kan St U, Manhattan, KS

Malloy, Sean, IRS, Holtsville, NY

Manatakis, Emmanuel K, U of Patras, Patras, Greece

Maneer, William S, Artronic Info Sys, New York, NY

Manolache, Nicolae C, Str Magi Ghita 7 Sect 8, Bucharest, Rumania

Maratta, James J, U of Cincinnati, Cincinnati, OH

Martin, John R, U of Saskatchewan, Saskatoon (S7N0W0), Sask Can

Masaitis, Ceslovas, Ballistic Res Lab, Aberdeen Proving Ground, MD

Mason, John B, Cambridge School of Weston, Weston, MA

Massironi, Massimo, via Varese, 142, (21047) Saronno, Italy

Masters, Thomas G, Computer Sci, Silver Spring, MD

Mc Gillivray, William R, Axter Machine, Mancelona, MI

McCallum, William A, Kan St U, Manhattan, KS

McClay, Douglas T, Rutgers U, NCAS, Newark, NJ

McCowan, Otis B, Belmont C, Nashville, TN

McDonough, J M, Prose, Los Angeles, CA

McKennon, Kelly D, Wash St U, Pullman, WA

McKeon, James P, Houston's, Portland, OR

Meitus, V Ju, Ukrainian Acad of Sci, (252207) Kiev (207), U S S R

Melvin, William R, Brown \& Root, Glen Rose, TX

Menas, Telis K, UCLA, Los Angeles, CA

Metcalf, Harold F, 3155 NE 36th Ave, Portland, OR

Meyer, W Weston, General Motors, Res Labs, Warren, MI

Meyn, Helmut, U of Erlangen-Nurnberg, (D-8520) Erlangen, Fed Rep Germany

Michelsohn, Marie-Louise, UCB, Berkeley, CA

Mikulecky, D C, Va Commonwealth U, Richmond, VA

Miller, Ronald R, Cal St U Hayward, Hayward, CA

Milne, Stephen C, Yale U, New Haven, CT

Milstein, G N, Uralskii St U, (620083) Sverdlovsk, U S S R

Miniowitz, Ruth, Israel Inst of Tech, Haifa, Israel

Minlos, R A, Moscow St U, Moscow, U S S R

Minsker, Steven, Rutgers U, Camden, Camden, NJ

Modeer, James R, U of Colo, Colorado Springs, CO

Montemayor, Jose J, U of Tex at Austin, Austin, TX

Montemezzi, Marco A, American U, Washington, DC

Moore, Robert H, Chicago Bd of Educ, Chicago, IL

Morel, Kathryn J, U of Tex at Arlington, Arlington, TX

Morgan, James Campbell, Snead St Jr C, Boaz, AL

Moritz, Elan, 405 E 56th St, Apt 3L, New York, NY

Morris, Howard C, City of Shreveport, Shreveport, LA

Moy, Allen, 4449 Broadway, Chicago, IL

Moye, Stephen Graybill, Box 6042, Boston, MA

Mueller, Dale E, U of Mont, Missoula, MT

Mullen, Michael S, Alamo Jr HS, Midland, TX

Murdock, James W, Nightingale-Bamford School, New York, NY

Mutlu, Moiz, Worcester County Natl Bank, Worcester, MA

Nariboli, Gundo A, Iowa St U, Ames, IA

Navarro Urgelles, Ramon M, U Pedagogico Maracay, Maracay, Venezuela

Nayak, M K, Flinders U, Bedford Park (5042), Australia

Nebb, J, OAO Corp, Beltsville, MD

Nebesny, Laurence Michael, Commodity Futures Trading Comm, New York, NY

Neimann, Albert A, Weapons Quality Eng Ctr, Concord, CA 
Nemzer, Daniel E, Ohio St U, Columbus, $\mathrm{OH}$

Newton, Theodore H, East Stroudsburg St C, East Stroudsburg, PA

Nielsen, Sigurd O, Symplexor, (DK-2840) Holte, Denmark

Niemuth, Jeffrey, Teledyne Brown Eng, Huntsville, AL

Noble, Ben, $\mathrm{U}$ of Wis, Madison, WI

O'Connor, John J, 10 Bryer Rd, Malden, MA

O'Mahoney, Rosalie, C of San Mateo, San Mateo, CA

Oconnell, James R Jr, Vought, Dallas, TX

Oertly, Alda F, 66 DeCatur Rd, Guilford, CT

Oertly, Frederick J, 66 DeCatur Rd, Guilford, CT

Oliker, Vladimir I, Temple U, Philadelphia, PA

Ooms, Alfons I, U Centrum Limburg, Limburg (3610), Belgium

Opelka, James H, Argonne Natl Lab, Argonne, IL

Oswald, Urs, Literargymnasium, (8037) Zurich, Switzerland

Page, David A, U of Ill at Chicago Circle, Chicago, IL

Pais, John, Webster C, St Louis, MO

Pallaschke, Diethard, U of Munster, Math Inst, Munster, Fed Rep Germany

Paneitz, Stephen M, 1021 Tennessee, Lawrence, KS

Pap, Maria, U of Windsor, Windsor, Ontario Can

Parker, George E, Emory U, Atlanta, GA

Parrish, Daniel M, 775 Waterloo Rd, Suffield, OH

Pasta, John R, NSF, Washington, DC

Patera, J, Math Res Ctr, Montreal, Quebec Can

Patten, Paul R, U of Okla, Norman, OK

Pearcy, Carey M, Busby Eng Const, Beaumont, TX

Peitgen, Heinz O, U of Bonn, (53) Bonn, Fed Rep Germany

Pennline, James A, Va Commonwealth U, Richmond, VA

Perez Carreras, Pedro, U of Valencia, Valencia (10), Spain

Playtis, Ann Scrandis, Swarthmore C, Swarthmore, PA

Prewitt, Judith M S, Natl Inst of Health, Bethesda, MD

Quinlan, Mary K, Trumbull HS, Trumbull, CT

Ramey, James T Jr, Francis Marion C, Florence, SC

Rasmussen, Carl Henry, $\mathrm{U}$ of Me, Orono, ME

Rauff, James Vernon, C of Lake County, Grayslake, IL

Raymond, Eric Steven, U of Pa, Philadelphia, PA

Redlin, Lothar H, McMaster U, Hamilton (L8S4K1), Ontario Can

Reese, Matthias F, Conrac, Alston Div, Duarte, CA

Reichel, Lee Charles, O'Brien \& Gere Engrs, Syracuse, NY

Reicher, Israel R, Baker \& Taylor, Somerville, NJ

Reitano, Robert R, John Hancock Mutual, Boston, MA

Reitwiesner, George W, 2201 Salisbury Rd, Silver Spring, MD

Renner, Richard R, 30850 Park Dr, Pepper Pike, OH

Richardson, Richard L, Battelle-Northwest, Richland, WA

Riegger, Charley, 345 N 3rd St, Apt 3, San Jose, CA

Rimmer, Russell, LaTrobe U, Bundoora (Vic 3083), Australia

Rinaldi, Leonard D, Rinaldi Data Processing Cons, Cranford, NJ

Roberts, Lawrence Gordon, U of British Columbia, Vancouver, Br Columbia Can

Rodino, Luigi, Inst Math Polytech, (I-10129) Torino, Italy

Romo, John Gilbert, Bee County C, Beeville, TX

Rose, David A, U of South Fla, Tampa, FL

Rosenbaum, Bruce, 172-40 133rd Ave, Jamaica, NY

Ross, Paul, 3233 W Dickens, Chicago, IL

Roullard, George P, Pacific Tele, San Diego, CA

Ruehr, Otto G, Mich Tech U, Houghton, MI

Ruess, W, U of Bonn, (53) Bonn, Fed Rep Germany

Rumore, Charles A, 9033 Contee Rd, Apt 301, Laurel, MD

Rumsey, Mary Ben, U of Ala in Birmingham, Birmingham, AL

Sack, Ira Howard, Canisius C, Buffalo, NY

Salles, Maurice, U of Caen, Caen, France

Sampson, Gary, SUNY, Ctr at Buffalo, Amherst, NY

Sands, Bill, U of Winnipeg, Winnipeg, Manitoba Can

Santoni, Larry J, 1520 Gregory, San Diego, CA

Sarabia, Jose, Inst U Politecnico, Barquisimeto, Venezuela 
Schechter, Eric, U of Chicago, Chicago, IL

Schechter, Martin T, Polytech Inst of New York, Brooklyn, NY

Schiller, Harvey Jack, 3343 Ainslie, Chicago, IL

Schmid, Jurg, U of Bern, (CH-3012) Bern, Switzerland

Schroder, M, Waikato U, Hamilton, New Zealand

Schroeck, Franklin E Jr, Fla Atlantic U, Boca Raton, FL

Seeling, Ronald L, U of Ky, Lexington, KY

Seiferas, Joel, Pa St U, University Park, PA

Sepehrnoori, Kamy, U of Tex at Austin, Austin, TX

Sficas, Yiannis G, U of Ioannina, Ioannina, Greece

Shafaat, A, 14 Churchill Ct, Apt 805, Woodlawn (Dartmouth) (B2X1N4), Nova Scotia Can

Shalen, Peter B, Rice U, Houston, TX

Shallon, Caroline R, Hughes Aircraft, Los Angeles, CA

Shapovalov, Nocolay N, Grimau St, 7/2, Cor 3, F66, Moscow (117036), U S S R

Shaw, Beryl I, Baruch C (CUNY), New York, NY

Shenk, Al, UCSD, La Jolla, CA

Shu, Tsao-Wen, 1009 W California Ave, Urbana, IL

Siddiqi, Rafat N, U de Moncton, Moncton, New Brunswick Can

Silva, Julio E, U d'Ottawa, Ottawa (K1N6N5), Ontario Can

Silverman, Richard Allan, $40 \mathrm{E} 9 \mathrm{th} \mathrm{St}$, Apt $12 \mathrm{H}$, New York, NY

Singer, Michael, Ohio St U, Columbus, OH

Sjoberg, Boris H, Abo Akademi, (20500) Abo (50), Finland

Smith, Thomas J, Manhattan C, Bronx, NY

Soifer, Alexandr Jr, Moscow St Pedogogical Inst, Moscow (G-435), U S S R

Stanilow, Gr, Bulgarian Acad of Sci, Sofia, Bulgaria

Stanton, Dennis W, U of Wis, Madison, WI

Stavre, Petre, U of Craiova, Craiova, Rumania

Steenken, Caroline M, Fayetteville St U, Fayetteville, NC

Stevanovich, Dushan, U of Ill at Chicago Circle, Chicago, IL

Stokes, Marion Jeffries, U of Richmond, Richmond, VA

Streit, Roy L, NUSC, NLL, New London, CT

Stutz, John K, SUNY, Ctr at Albany, Albany, NY

Swisher, William K, Clermont General \& Tech C, Batavia, OH

Taylor, Alan D, Union C, Schenectady, NY

Thomas, G, U of Western Ontario, London (N6A5B9), Ontario Can

Thomas, Lawrence E, U of Va, Charlottesville, VA

Thornton, Edward G Jr, 120 Evergreen Dr, East Providence, RI

Thorsell, Ulf, Solhaga Sjnkhem, (28040) Shanes Fagerhult, Sweden

Torres, Ennodio J, U Ctr Occidental, Barquisimeto, Venezuela

Trushin, David, 1535 E Stonehenge, Sycamore, IL

Tucci, Ralph P, U of Wis-Milwaukee, Milwaukee, WI

Van Hai, Nguyen, 4327 Longene Ct, Louisville, KY

Van Rees, G H John, U of Waterloo, Waterloo (N2L3G1), Ontario Can

Vasilescu, F-H, Inst of Math, Bucharest, Rumania

Wang, Albert Tso-Ming, U of Tenn, Knoxville, TN

Wang, Cecilia Y, Ariz St U, Tempe, AZ

Wefelscheid, Heinrich, U of Duisburg, (4100) Duisburg, Fed Rep Germany

Weidner, Ralph, U of Tenn, Knoxville, TN

Weiss, David E, Western Wash St C, Bellingham, WA

Welch, R L W, U of South Fla, Tampa, FL

Wesley, Eugene, Wayne St U, Detroit, MI

Whitt, Ward, c/o M M Sacks, 40 Deer Hill Dr, Hohokus, NJ

Wilkerson, Clarence W, U of Pa, Philadelphia, PA

Williams, James Eugene, 1436 W St NW, Washington, DC

Williams, Samuel T, B F Goodrich, Brecksville, OH

Williams, Stephen A, Wayne St U, Detroit, MI

Wilson, Steven J, Argonne Natl Lab, Argonne, IL

Winternitz, Pavel, U de Montreal, Montreal (101), Quebec Can

Witzgall, Christoph, NBS, Washington, DC

Wong, Kin Chung, U d'Ottawa, Ottawa (K1N6N5), Ontario Can

Woy, Jean L, Dun-Donnelley Publ, New York, NY

Wynn, Gregory, Ill Inst of Tech, Chicago, IL

Wynn, Varona L, Clark C, Atlanta, GA 
Yannacopoulos, Dimitrios S, NYU, New York, NY

Zafarani, Jafar, U of Isfahan, Isfahan, Iran

Zaicev, D I, Ukrainian Acad of Sci, Kiev, U S S R

Zalesak, Rudolph P Jr, 2000 Pearl St, Austin, TX

Zayed, Ahmed I, U of Wis-Milwaukee, Milwaukee, WI

Zorzitto, Frank A, U of Waterloo, Waterloo (N2L3G1), Ontario Can

Zurbenko, I G, Moscow St U, Moscow (117234), U S S R

Zweifel, Paul F, VPI \& SU, Blacksburg, VA

\section{Membership Under Reciprocity}

Australian Mathematical Society: Hickman, J L; Ozanne, Kenneth W; Parrott, David

Deutsche Mathematiker-Vereinigung e. V.: Kleinstuck, Gert; Leeb, Klaus; Nguyen, Xuan Xanh; Otte, Michael; Schmid, Peter P

Indian Mathematical Society: Gabbay, Dov M; Ramamurthi, V S; Rao, N

Mahalingeshwara; Tamuli, B K

Íslenzka Staerdfraedafélagid: Briem, E

Israel Mathematical Union: Bshouty, David; Eliahu, Zini; Feder, Moshe; Har'el, Zvi; Milman, V D; Zalik, R A

Korean Mathematical Society: Han, Chul-Soon

London Mathematical Society: Lake, John; Lehrer, Gustav I; Mc Connell, John C; Tomaras, Alexander; Vamos, Peter

Mathematical Society of Japan: Imai, Hideo; Jun, Byung Moon; Koriyama, Akira; Yaku, Takeo

Norsk Matematisk Forening: Krogstad, Harald E

Polskie Towarzystwo Matematyczne: Kucharzewski, M; Macedonska-Nosalska, Olga

Real Sociedad Matemática Española: Bombal, Gordon F

Schweizerische Mathematische Gesellschaft: Kirchbraber, Urs

Sociedad Matemática Mexicana: Ontiveros, A Jorge; Ruben, Flores Espinoza

Sociedade Brasileira de Matemática: Milla Miranda, M; Neto, Artur Milhomem

Société Mathématique de Belgique: Ballieu, M A G; Dentant, E-J

Société Mathématique de France: Bermond, J-C; Launay, Didier M; Loday, Jean-Louis;

Raynaud, Jacques F; Seydi, Hamet; Sjostrand, Johannes N; Soto Andrade, Jorge

Suomen Matemaattinen Yhdistys: Staffans, Olof J

Svenska Matematikersamfundet: Boo, Per-Anders

Unione Matematica Italiana: Arzarello, Ferdinando; Soardi, Paolo Maurizio

Wiskundig Genootschap: Van Den Boogerd, J G; Van Mill, Jan

\section{Nominees of Institutional Members}

Abilene Christian Univ: Smallwood, John L Jr

Auburn Univ, Auburn: Cox, Gregory V; Jordan, Diane; Lane, Keith Whiteside; Phelps, Kevin T

Ball St Univ: Blinn, Lucille K; Dillon, Robert A

Boston Univ: Druecker, Robert P; Katz, Elliot I; Rush, John David; Smith, Michael B

Brandeis Univ: Drager, Lance D; Hulbert, Douglas S; Leichter, Jerrold; Sato, Nobuyuki A

Brigham Young Univ: Adams, Richard T; Stark, David B; Wu, Edward

Brock Univ: Heinrich, Harald W; Msemakweli, Anthony S; Parent, Leonard G; Waldock, T-Jay

Brown Univ: Avellar, Cerino E; Coelho, Jose R; Doellman, John L; Fleri, Edward C; Frolow, Igor; Martinez-Amores, Pedro; Reber, Douglas C; Sayward, Frederick G; Somolinos, Alfredo S; Tsen, Fu-Shiang P

Calif Inst of Tech: Coutsias, Evangelos Athanassios; Fokas, A; Hagan, Patrick Shawn; Huffman, Michael D; Kastanas, Ilias G; Lentini, Marianela; Mueller, James R; Shapiro, Helene

Calif Polytech St Univ: Adorno, David Samuel; Davis, Christine L; Stoutenborough, Ryan L; Warrack, A Giles

Calif St Univ, Fullerton: Cartwright, Gayle S; De Land, Paul N; Duhme, Cheryl D; Kroh, Steven John

Calif St Univ, Hayward: Brandt, Roger H; Cox, David M; Thompson, Joel S; Van Der Byl, Baldwin $\mathrm{J}$ W 
Calif St Univ, Long Beach: Downey, Stephen J; Himes, John; Rizzardi, Frances I;

Roussere, Phillip; Sundell, Jan

Calif St Univ, Sacramento: Missel, Colin H; Pieracci, Laurie

Calvin Coll: Dejong, Jack C; Thomasma, Timothy D; Van Baak, Thomas L

Carleton Univ: Ivanoff, B Gail; Kulperger, Reginald John; Mahendrarajah, A; Ramanath,

$\mathrm{M}$ V S

Case Western Res U: Franks, Eugene W; McKelvey, Richard H

Central Michigan Univ: Brown, Anne E; Carson, Judy L; Turski, Alan R

Claremont Colleges: Abrahamson, David L; Christ, F Michael; Cleland, Bruce Alan; Farris, Frank A; Ju, Jean H Wang; McCartney, Philip W; Scott, David W

Clemson Univ: Benz, Stephen Lewis; Edwards, Thomas B; Johnson, S L; Jones, Albert F; Lewis, Jerome Luther; Newruck, Frederick C; Potter, Robert A; Tosch, Thomas J

Cleveland St Univ: Westgate, Gregory A

Columbia $U$ : Bourin, Claude M; Elman, Howard; Feighn, Mark E; Heifetz, Dennis B; Hobelman, James Bryant; Hrabowski, Jan; Littleford, Alan; Ramakrishnan, Dinakar; Riera, Gonzalo; Rose, Julian; Thomas, B Lupo; Valenza, Robert J

Concordia Univ: Chang, Stella Ann Chi-Hsing; Chassagne, Stanley R; Frajberg, Laine; Trivellizzi, Mariassunta

Dalhousie Univ: Edelstein, Leah; Gray, Greg; Lever, David B; Rai, Rajendra K; Scott, Paul James; Tingley, Daryl W

Emory Univ: De Caux, Peter A; Peeples, Donald R

Fairleigh Dickinson U, Teaneck: Sarkisian, Richard G

Federal City Coll: Brown, Brenda E; Chloe, Ronald 1; Edwards, Terrence A; Reynolds, Arnold James

Florida Atlantic Univ: Bastida, Vicki C; Boitet, Val C; Miller, Kathleen Shovan; Pallack, Robert Scott

Florida St Univ: Chen, Kuo-Chang; Kutter, Mary Y; Morrison, Tim H; Pearson, Stan C; Rimbey, Scott E

Georgia St Univ: Graham, Vickie L

Grambling St Univ: Woodard, Lawrence

Harvard $U$ : Goss, David $\mathrm{M}$

Haverford Coll: Alperin, Roger Charles

Herbert H Lehman Coll, CUNY: Rocchi, Albino M

Howard Univ: Hill, Stacy Douglas; Smith, Sandra

Hunter Coll, CUNY: Bouckris, Arlene; Kreiter, Gilead Abraham J Y

Illinois Inst of Tech: Caldwell, Janet Y; Lenard, Anthony; Levesque, Claude; Reichel, Paul G; Snyder, Richard D

Indiana Univ at Bloomington: Brown, Patrick J; Brunson, Barry W; Carlson, Mark A; Casero, Emilio; Christenson, Peter David; Chuang, Tson-Chih J; Collins, Clyde Edwin; Dickelman, Gary J; Drzal, Keith A; Grannemann, Richard S; Habegger, Nathan B; Howell, Kenneth B; Jones, Thomas Charles; Keenan, Daniel MacRae; King, John J; Purdy, Dale R; Rallis, Nancy E; Wright, Steve J

Kalamazoo Coll: Adams, Gregory T; Foote, Robert L

Kansas St Univ: McHugh, Mike; Perez, Jose Jesus; Schultz, J H

Knox Coll: Ong, Kho Sian; Wickart, William D; Zimmerman, Jay J

Kutztown St Coll: Newman, Barbara $\mathrm{H}$

Lake Forest Coll: Kash, Michael M

Long Island Univ, Brooklyn Ctr: Andrews, Rennie Lawrence; Mendez, Nancy A

Louisiana St Univ, Baton Rouge: Kramer, Robert J; Scott, Mark W

Massachusetts Inst of Tech: Anderson, Robert Joseph; Mendoza, Ramon; Uhlmann-Gunther McMaster Univ: Andrus, Dennis; Bradley, John Scott; Laxton, James A

Miami Univ, Oxford: Gilmore, Stephen V; Krafcik, Thomas M

Millersville St Coll: Fettes, Susan E; Hitchman, Ronald G; Moser, Susan C; Prestwood, John C

Monmouth Coll: Kloeppel, Peter K

Murray St Univ: Fraser, Robert Wayne; Kaelin, David Lawrence; Meadors, Luke Allen; Shelton, James E

New Mexico St Univ, Las Cruces: Engebos, Bernard F; Rojo, Oscar

New York Univ, Courant Inst: Bassein, David A; Baumbach, Jim; Bryant, Charles A; Caflisch, Russel; Chang, I-Chung; Clifford, Jane E; Coppage, Samuel F; Couet, Benoit; Digri, Vincent J; Dozier, Lewis B; Echeverria, Pedro E; Ferreira, Wilson Castro; Gogolak, Carl V; Goldfinger, Helen Susan; Klainerman, Sergio L; Leibovitch, Aizik; Levermore, Charles David; Levi, Mark; Lieb, Mark; Lucardi, Linda Ann; McFadden. 
Geoffrey B; Michener, Carol Bolomey; Ni, Wei-Ming; Raifaizen, Claude H; Rapoport, David; Rappaport, Jack M; Rennert, Philip Murray; Robins, Charles Matthew; Sanz, Jose M; St Vincent, Michael; Stephenson, George; Strumolo, Gary Steven; Sylvester, John $\mathrm{H}$

Northeastern Illinois Univ: Gress, Charles R

Northern Illinois Univ: Bahl, Satish K; Harrington, Richard A; Peterson, David Lawrence; Plass, Susan Lee; Pomering, Raymond H; Portegys, Thomas E; Williams, Judith A

Northwest Missouri St Univ: Boswell, Paula S; Cornell, Linda M; Hayes, Gary E; Shoebrook, Mary Ann

Northwestern St Univ: Matovsky, John C; Prasitsak, Prapa Poowaton

Northwestern Univ: Beck, Randal S; Finnigan, Skip; Harris, Kathleen A; Hurley, Michael G; Kleinerman, Samuel; Kuchenbecker, William G; Patterson, Denise I; Reese, Robert Boyd; Secada, Walter G; Terrell, William J; Unell, Alan D; Weakley, William Douglas; White, Denis A

Ohio St Univ, Columbus: Dinitz, Jeffrey H; Fiedler, Joseph R; Kahn, Jeffry Ned; Kirwin, Peter J; Roth, Robert L Jr; Waters, Carl A

Ohio Univ at Athens: Grabner, Gary Clem; Konrad, Michel David; Norman, Daniel C; Sagong, Seok

Old Dominion Univ: Donahoe, Irene C

Oregon St Univ: Clark, Robert T; Dranchak, John J; Fredricks, Gregory A; Greenfield, Gary R; Kelley, Thomas E; Lancaster, Kirk E; McCay, Brian M; Ng, Mary J; Ray, Craig Anthony; Stynes, Martin

Pace Univ, New York: Buckley, Fred

Pacific Lutheran Univ: Critchlow, Susan C; French, Susan L; Trautmann, Rolf; Upton, Kevin Lee

Plymouth St Coll: Dearborn, Barbara S; Ferland, Keith R; Miller, Beth A

Portland St Univ: Josephson, Linda Susan; Perkins, James L; Serven, Robert J; Twombly, Evan E

Princeton Univ: Gomez-Mont, Xavier Avalos

Purdue Univ, Calumet Campus: Baron, Eugene W

Queen's Univ: Bougas, I-John; Halfyard, Clayton W; Kovacic, Frank A; Novikoff, Gordon R; O'Shea, Donal B; Yu, Kenneth Puikan

Queens Coll, CUNY: Strauss, Ronald M

Rice Univ: Yingst, $\mathrm{H}$ David

Rockford Coll: Klotz, Jeffrey Owen; Lampman, Sandy L; McFadden, Thomas B

Sam Houston St Univ: Allbritton, Gay Janelle; Casey, Helen Maryse; Cross, James H II: Shimada, Mitsuaki

Shippensburg St Coll: Jones, Frank Thomas; King, Eric E

Simon Fraser Univ: Raphael, Claudette; Varma, B N

Southern Ill U, Carbondale: Becker, Leigh Carl; Lien, Ming-Chiao; Shoaff, William D; Wang, Clement S L

Southwest Texas St Univ: Habluetzel, Jackie; Keepers, Lonnie L

Southwestern at Memphis: Eastham, Jerome F

St Olaf Coll: Lustgarten, Paul C

Stockton St Coll: Mills, Charles F; White, Jeff W

SUNY, Coll at Cortland: Mosbo, E Paul

SUNY. Coll at Geneseo: Dussault, David A

SUNY. Ctr at Albany: Falconieri, Thomas R; Grice, Judith A; Hosmane, Balakrishna Sadashey; Law-Kuok-Kouong; Ramaroson; Francois X; Ugolik, Wayne R

SUNY, Ctr at Binghamton: Black, David L

SUNY. Ctr at Buffalo: Chen, Wu-Young; Dai, Loa-Sheng; King, Fenn; Rosenthal, Kimmo I; Tamhankar, Madhav V; Yang, Shi-Nine

SUNY, Ctr at Stony Brook: Freeman, James S

Syracuse Univ: Forster, Jean Frieda

Tennessee Tech Univ: Jessee, David W; Liu, Han-Chu Albert; Torgerson, Claudia Gail

Texas $A$ \& I U, Kingsville: Johnson, David W; Kay, Mary Heck; Wyche, Sandy Amoros

Texas $A$ \& $M$ Univ: Chow, Jeff; Cole, Herman D; Feiring, Bruce R; Peterson, Douglas L; Podisuk, Maitree; Shen, Li-Chien; Slinker, Steven P; Smith, Roger R; Whitehead, Jennifer; Zeller, Mike

Texas Tech Univ: Kern, Dennis Matthew; Neil, Charles $\mathrm{H}$

$U$ of Arkansas at Fayetteville: Mulkey, Gary R

$U$ of Ill at Urbana-Champaign: Dougan, Robert E Jr; Sonheim, Jeffrey

$U$ of North Dakota, Grand Forks: Gunvalson, Michael C; Smith, Steven G 
U of Puerto Rico, Rio Piedras: Bird, Aida Canals; Rodriguez de Andueza, Priscilla; Schmitt, Paul W

$U$ of Southwestern Louisiana: Broussard, Kathleen Ann; Norwood, Frederick H

$U$ of Wisc, Madison: Anderson, Eugene R; Arratia, Richard Alejandro; Bassett, Edward M; Bassignana, Daniel F; Chen, Goong; Compton, Kevin J; Dechansreiter, Max; Koh, Hock-Lye; Leys, John; Neidhardt, Arnold L; Olson, Douglas W; Parker, Jeffrey D; Parsons, Lee; Price, Roderick A; Rentmeesters, Elizabeth; Sentoff, Stephen; Sirota, Warren M; Sternfeld, Robert William; Sundberg, Carl; Talmage, P Gregory; Ullrich, David C

Univ de, Costa Rica: Abarca, Isaac; Alvarado Jimenez, William; Duate. Asdrubal; Estrada N, Richardo

Univ de Montreal: Antonius, Wedad; Baddou, Jamal Addin; Dubeau, Francois; Hamel, Serge; Lassonde, Marc; Penon, Jacques Henri

Univ of Akron: Garzia, Mario Ricardo; Mikolaj, John Joseph Jr; Okonieski, Deborah Ann; Ranaldic Paolo

Univ of Alabama-Birmingham: Huey, Laura M

Univ of Alabama-Huntsville: Cashion, Robert C; Henderson, Jean F; McCune. Garey K

Univ of Alberta: Ehrhardt, Edwin G; Manetto, Luis; Mysore, Sudhesh Malliah; Tosh, Donald $\mathrm{H}$

Univ of Calif, Berkeley: Bass, Richard F; Clark, Stephen A; Cosner, George C; Davis, Glenn H; Edwards, Harriet C; Fall, Thomas C; Faran, James J; Green, Philip; Harer, John L; Harman, Jonathan W; John, Thomas; Kaplan, Robert Frank; Krumme, David W; Lee, Calvin B; Lugo, Gabriel Guillermo; Margolis, Stuart W; Merzel, Jonathan L; Ochoa, Juan C; Ong, Boon-Hua; Pearce, Jon; Pieniazek, Lester; Ran, Ziv; Rassias, George M; Shifrin, Theodore; Siemon, Michael L; Straubing, Howard; Stroik, John Richard; Tam, Kin On; Wallwork, David; Wei, S Walter; White, Ray Richard; Williams, Daniel Arthur; Wilson, Stephen Earl

Univ of Calif, Irvine: Barbee, Terry William; Brennan, Terry J; Christenson, Peter G; Murufas, Roderic; Patterson, Donald A

Univ of Calif, Los Angeles: Dickinson, Richard P

Univ of Calif, San Diego: Galloway, Gregory J; Glover, Joseph; Mitro, Joanna B; Oldham, Frank Ernest; Pourciau, Bruce Hunter; Shapiro, Joni A; Smith, Cory B; Sorensen, Danny C; Sweetser, Theodore H III; Wachs, Michelle L

Univ of Calif, Santa Barbara: Andrews, William W; Chollet, John Paul; Collier, David C; Klein, David; Konas, Gary; Kuo, Morgan M C; Lee, Tan-Yu; McCullough, Dick; Mitchell, James C; Orlandos, Peter Anastase; Rinne, Dan C; Shen, Nien-Tsu; Verrill, Steve $\mathbf{P}$

Univ of Chicago: Arnold, Douglas N; Bodeau, Deborah J; Ginali, Susanna; Hermann, Diane L; Iannaccone, Laurence R; Katzenstein, Leo Rex; Lin, Der-Long; Sze, David Y; Tiwari, Sanjay; Williams, Eric Devon; Yu, R H

Univ of Delaware: Hangelbroek, Rutger $\mathrm{J}$

Univ of Denver: Armstrong, Judith Anne; Casstevens, Harold T II: Harvill, Demian

Univ of Florida: Chen, Raymond

Univ of Hartford: Groth, Philip H; Lagosz-Sinclair, Donald B

Univ of Houston, Houston: Bo-Linn, Cheevon; Brewerton, Dottie C; Dennis, Thomas B; Kaufman, Susan Lamb; Marsh, Dorothy Sherling; Redner, Richard A

Univ of lowa: Downing, David J; Haack, Joel Knight; Hansen, Kristina Dale; McAsey Michael J; Merrill, Stephen J; Seltzer, Stanley E; Tudor, David W

Univ of Kansas: Atkins, David L; Bartscher, William J; Chang, Shu-Jung; Feil, Todd H; Makin, James R; Schwartz, Niels C; Shimpi, Ashok

Univ of Kentucky: Euler, Russell Nelson; Klemesrud, Bruce H; Leverenz, Christine R; Saylor, Annie V; Van Niewaal, Calvin R; Wright, Ronald H

Univ of Louisville: Bledsoe, Margaret Randolph; Friedberg, Howard Alan; Hohman, Kenneth F; Mudd, Anne Chestney

Univ of Maine, Orono: Rich, David; Treger, Jerold R; Wiken, Randall T

Univ of Massachusetts, Amherst: Derooij, Alfred; Gold, Benn; Lukesh, Gordon W; Moore, Thomas T; Rice, Richard E

Univ of $M d$, Baltimore County: Brill, Clark R; Doyle, Patricia J

Univ of Michigan, Flint: Greene, Steven Jay; Leonard, Jon

Univ of Minnesota-Minneapolis: Chiang, Tzuu-Shuh; Cranston, Michael Craig; Guelich, Julie A; Hamlin, Dennis D; Hummel, Robert A; Madden, Dick; Phillips, Daniel; Sprecher, Richard C; Taranto, Mark Allen; Tlougan, Donald J; Unguriano, Constantino; Yuh, Yih-Shyh 
Univ of Missouri, Columbia: Elaydi, Saber N; Helsabeck, Henry C; Milne, Kathleen M

Univ of Missouri, Rolla: Fitzpatrick, William J

Univ of $N C$ at Chapel Hill: Branton, Michael Gerald; Chambless, Lloyd E; Fennell, James G; Koby, Marilyn Frances; Kroah, William T; McDuffee, Robert B; Patterson, Samuel E; Rhaly, Henry Crawford Jr; Webb, David L; Wei, Tung-Sheng

Univ of $N$ C at Charlotte: Maier, Joseph E; Walker, Joey

Univ of $N C$ at Greensboro: Heatherly, David L

Univ of Nevada, Reno: Ash, Edward Leslie; Coleman, Kenneth

Univ of New Orleans: Bigelow, Patrick William; Soileau, Peggy Lynn

Univ of Oklahoma: Naylor, Lawrence W; Roark, Charles W

Univ of Oregon: Bhattaraj, Hom Nath; Fernandez, James William; Keef, Patrick W

Univ of Pitsburgh, Pittsburgh: Amit, Rafi; Cohen, Jeffrey Mitchell; Cox, Catherine Marie; Nelson, Robert R; Sastry, N S Narasimha; Sinha, Bimal Kumar; Tan, Suan-Boon; Tod, Paul; Urbano, Donnie M

Univ of Rochester: Deangelis, William J; Ellis, C Lane; Goncalves, Daciberg Lima; Roy, Dev Kumar

Univ of Tennessee, Knoxville: Cain, William D; Gupta, Ritu R G; Hassell, David F; Knauer, Linda M; Preston, Donald K; Watkins, Joseph C III

Univ of Texas at Austin: Goth, John A; Shirley, Frank T

Univ of The West Indies: Aub, Martin R; Henry, Leighton Lloyd; Mair, Bernard A

Univ of Toledo: Bixler, J Patrick; Bulusu, Somayajulu; Duvvuri, K Rao; Nagel, Nancy W

Univ of Virginia: Cunningham, Charles D Jr; Hardin, Clyde D Jr; Herring, Sandra; Hill, Larry Thomas; Tucker, Richard

Univ of Washington: Baum, Lawrence; Baxter, James A; Fitzpatrick, Simon Peter; Hubert, Steven L; Meaney, Christopher; Moerk, Charles; O'Halloran, Joyce; Odgers, Christopher John; Pruiksma, Glenn J

Univ of Waterloo: Anstee, Richard Paul; Cargill, Thomas Alan

$V P I$ \& SU: Dymacek, Wayne Marshall; Eklund, Anthony D

Wake Forest Univ: Carter, Philip L; Dobyns, Mary Susan; Sulek, Joanne M

Washington St Univ: Notestine, Ronald D; Rennie, Michael W

Wayne St Univ: Chisholm, Michael Ernest; Jokela, David L; Lhota, Frank J; Ng, Kit Ying; Oversteegen, Lex G; Seyfarth, Charles Anthony; Snabb, Thomas E

Wesleyan Univ: Pelland, David S; Retta, Teklehaimanot

West Virginia Univ: Obermann, Carol L; Swyter, Timothy C

Western Michigan Univ: Barstow, Timothy S; Boucher, Clarence E Jr; Burns, David Peter; Chau, Wing Cheong

Western Washington St Coll: Hickenbottom, Paul L; Olson, John E; Strand, Richard A

Winthrop Coll: Davis, Jerry W

Xavier Univ: Schumacher, Donald W

Yale Univ: Anderson, Robert M; Barnes, Donald W; Breidbart, Seth I; Cameron, John A; Chandra, Rati; Chastkofsky, Leonard; Eddy, William F; Ehrhardt, Richard; Elton, John H; Estey, Arthur S; Friedman, Herman P; Herman, Franklin B; Hogben, Leslie; Jungster, Jerry J; Maeda, Michie; Record, Stephen E; Reiss, Steven P; Schreiber, Robert S; Schwager, Steven J; Seeley, Craig D; Spencer, Bruce; Thomas, Annie

Yeshiva Univ: Ehrlich, Susan Cheryl; Press, Claudia T; Zahavy, Reuvain

\section{INSTITUTIONAL MEMBERSHIP}

Boston University, Boston, MA

California State Polytechnic University, San Luis Obispo, CA

Concordia University, Sir George Williams Campus, Montreal, Quebec, Can

Federal City College, Washington, DC

Grand Valley State Colleges, Allendale, MI

Indiana University/Purdue University at Indianapolis, Indianapolis, IN

Plymouth State College, Plymouth, NH

Stockton State College, Pomona, NJ

SUNY, College at Cortland, Cortland, NY

University of Akron, Akron, $\mathrm{OH}$

University of Texas at El Paso, El Paso, TX

University of Western Ontario, London, Ontario, Can 